\title{
The Yamabe invariant of simply connected manifolds
}

\author{
Jimmy Petean \\ Max-Planck Institut für Mathematik, \\ Bonn, Germany
}

\begin{abstract}
Let $M$ be any simply connected smooth compact manifold of dimension $n \geq 5$. We prove that the Yamabe invariant of $M$ is nonnegative. This is equivalent to say that the infimum, over the space of all Riemannian metrics on $M$, of the $L^{n / 2}$ norm of the scalar curvature is zero.
\end{abstract}

\section{Introduction}

A classical problem in differential geometry is the determination of the family of functions that can be obtained as the scalar curvature of a Riemannian metric in some fixed smooth manifold $M$. As one might expect, the features of the problem in low dimensions are very different from the high-dimensional case. The classical Uniformization Theorem assures that any Riemannian metric on a 2-dimensional manifold is conformal to a metric of constant scalar curvature (in dimension two, this is the same as constant sectional curvature). For a compact surface, the Gauss-Bonnet formula then shows that the sign of such a constant is determined by the topology of the surface. Moreover, if we restrict to metrics of unit volume, then the value of the constant is completely determined by the topology (it is $4 \pi \chi$, where $\chi$ is the Euler characteristic of the surface).

In higher dimensions, we can still deform any metric to a metric of constant scalar curvature. Namely, given any Riemannian metric $g$ on a compact smooth manifold $M$, there is a metric conformal to $g$ which has constant scalar curvature. This is the well-known Yamabe problem. Yamabe first 
stated this result in 25], but his proof was not correct, as pointed out by Trudinger in [24]. The argument was completed in several steps by Trudinger [24], Aubin [3] and Schoen [20]. Note that in dimensions greater than two the obstruction to the existence of metrics with a determined sign is much weaker. For instance, every compact manifold of dimension at least three admits a metric of constant negative scalar curvature. There are well-known obstructions, though, to the existence of metrics of positive or vanishing scalar curvature.

If a function is expressed as the scalar curvature of a metric on a manifold $M$, any positive multiple of the function can be obtained by rescaling the metric. In order to avoid these "trivial" variations, and therefore to get a meaningful measure of the possible "size" of the scalar curvature function, it is reasonable to restrict to metrics of unit volume. This was first considered by O. Kobayashi in [8], where he introduced what we will call the Yamabe invariant of a compact smooth manifold $M$. First consider a fixed conformal class of metrics $\mathcal{C}$ on $M$, and let the Yamabe constant of $(M, \mathcal{C})$ be

$$
Y(M, \mathcal{C})=\inf _{g \in \mathcal{C}} \frac{\int_{M} s_{g} d v o l_{g}}{\left(\operatorname{Vol}_{g}(M)\right)^{2 / n}} .
$$

The solution to the Yamabe problem is precisely achieved by showing that this infimum is always attained by a smooth metric, which necessarily has constant scalar curvature. The Yamabe invariant is then defined by

$$
Y(M)=\sup _{\mathcal{C}} Y(M, \mathcal{C}),
$$

where the supremum is taken over all conformal classes of metrics on $M$. Note that this invariant is also frequently called the sigma constant of $M$ [21].

Note that the invariant is readily computable in dimension two from the Gauss-Bonnet formula. In dimension three, the computation of the invariant (of manifolds for which the invariant is non-positive) would follow from Anderson's program for the hyperbolization conjecture [1]. Computations of the invariant in dimension four have been carried out by LeBrun in [11]; he computed the invariants of all compact complex surfaces of Kähler type which do not admit metrics of positive scalar curvature. See also [5, 12, 17] for other computations of the invariant in dimension four. 
We will be concerned in this paper with simply connected manifolds of dimension greater than four. The study of manifolds which admit metrics of positive scalar curvature has proved to be very interesting and deep. See for instance the work by Gromov and Lawson [四], Schoen and Yau [19] and Stolz [22]. In this last paper, it is completely determined which simply connected compact manifolds of dimension greater than four admit metrics of positive scalar curvature. Recall that the Yamabe invariant of $M$ is positive if and only if $M$ admits a metric of positive scalar curvature (see for instance [21]).

We will prove:

Theorem 1 : Every simply connected smooth compact manifold of dimension greater than four has non-negative Yamabe invariant.

Note that for a manifold $M$ which does not admit positive scalar curvature metrics, the Yamabe invariant can also be computed by the formula

$$
Y(M)=-\inf _{\mathcal{M}}\left(\int_{M}\left|s_{g}\right|^{n / 2} \text { dvol }_{g}\right)^{2 / n}
$$

where $\mathcal{M}$ is the space of all Riemannian metrics on $M$ (see for instance [11).

It is also well-known that if a compact manifold (of dimension at least three) admits a metric of positive scalar curvature then it also admits a scalar-flat metric. We can therefore rephrase the previous theorem as:

Corollary 1 : For every simply connected smooth compact manifold $M$ of dimension greater than four,

$$
\inf _{\mathcal{M}} \int_{M}\left|s_{g}\right|^{n / 2} d v o l_{g}=0 .
$$

It follows from these results that dimension four is quite exceptional in terms of the Yamabe invariant. Note that Theorem 1 is obviously true in dimension two and clearly expected to be true in the 3-dimensional case (either from the Poincare Conjecture or from Anderson's program for the Hyperbolization Conjecture [1]). But it is definitely not true in dimension four (see the work of LeBrun in [10, 11]); moreover, it seems likely to be the case, from LeBrun's computations, that the generic simply connected compact four-manifold has strictly negative Yamabe invariant. 


\section{The Spin Cobordism Ring}

The aim of this section is the proof of Theorem 2 below. We will need to recall some basic facts about the spin cobordism ring. Details can be found in [9, 23]. Of course, we will need to consider in this section manifolds with boundary. And hence, in this section, we will call a manifold $X$ closed if it is compact and without boundary (in the other sections we always assume that the manifolds have no boundary).

For a spin manifold we will mean a smooth oriented manifold with a fixed spin structure on its tangent bundle. Let $X$ be a spin manifold with boundary. The spin structure on $X$ induces a canonical spin structure on the boundary of $X$ (see [14]), and two closed spin manifolds $X_{1}$ and $X_{2}$ of dimension $n$ are called spin cobordant if there is a compact spin manifold $X$ (of dimension $n+1$ ) so that $\partial X$ is, as a spin manifold, the disjoint union of $X_{1}$ and $-X_{2}$ (the minus meaning that the orientation is reversed). The set of equivalence classes of $n$-dimensional closed spin manifolds under this relation is called the $n$-dimensional spin cobordism group, $\Omega_{n}^{\text {Spin }}$. It is an Abelian group, with the sum given by the disjoint union or equivalently by the connected sum (if $X$ and $Y$ are connected spin manifolds, then $X \# Y$ inherits a spin structure so that $X \# Y$ is spin cobordant to the disjoint union of $X$ and $Y$ ).

Throughout this section we will denote by $[X]$ the class of the closed spin manifold $X$ in the spin cobordism group.

The product of manifolds gives a ring structure to $\Omega_{*}^{\text {Spin }}$. This is called the spin cobordism ring. Its structure has been determined by D.W. Anderson, E.H. Brown and F.P. Peterson in [2]. It is proved there that two closed spin manifolds of dimenion $n$ are spin cobordant if and only if they have the same Stiefel-Whitney and KO characteristic numbers. A particular example of these characteristic numbers, which will play an important role in this article, is the $\alpha$-homomorphism,

$$
\alpha: \Omega_{*}^{S p i n} \rightarrow K O_{*}(p t),
$$

first introduced by Atiyah and Milnor (see [14]). It is important for us that $\alpha$ is a ring homomorphism; i.e. for any connected closed spin manifolds $X$ and $Y, \alpha[X \# Y]=\alpha[X]+\alpha[Y]$ and $\alpha[X \times Y]=\alpha[X] . \alpha[Y]$.

Now recall that $K O_{n}(p t)$ vanishes for $n=3,5,6$ and 7, while $K O_{1}(p t)$ and 
$\mathrm{KO}_{2}(p t)$ are isomorphic to $\mathbf{Z}_{2}$, and $\mathrm{KO}_{4}(p t)$ and $K \mathrm{O}_{8}(p t)$ are isomorphic to $\mathbf{Z}$. Recall also that multiplication by a generator of $K O_{8}(p t)$ gives an isomorphism between $K O_{n}(p t)$ and $K O_{n+8}(p t)$. Moreover, $\alpha$ is an extension of the $\hat{A}$-genus in the sense that after picking suitable generators of $K O_{8 k}$ and $K O_{8 k+4}, \alpha$ is exactly the $\hat{A}$-genus in dimensions $8 \mathrm{k}$ and one half of the $\hat{A}$-genus in dimensions $8 \mathrm{k}+4$.

The homomorphism $\alpha$ plays a central role in the study of the scalar curvature of compact spin manifolds. Hitchin proved in [6] (generalizing the now classical work of Lichnerowicz [13]) that if the compact spin manifold $X$ admits a metric of positive scalar curvature, then $\alpha[X]=0$. The converse of this result is also true for simply connected manifolds. It was conjectured by Gromov and Lawson in [4], and proved in different cases by Gromov and Lawson [4], Miyazaki [15], Rosenberg [18] and finally (in general) by Stolz [22]. More precisely, Stolz proved that any cobordism class in the kernel of the homomorphism $\alpha$ can be represented by a connected spin manifold which admits metrics of positive scalar curvature (the result then follows from the arguments in [4]). Similarly, to prove Theorem 1, we will need to show that every element in the spin cobordism groups can be represented by a manifold with non-negative Yamabe invariant. We will prove this statement for every dimension in Theorem 2, although we will only need the result for dimensions greater than four. We will prove Theorem 2 now and then use it to prove Theorem 1 in the following section.

We will need to use the following obvious fact:

Lemma 1 : Suppose that $M$ and $N$ are closed smooth manifolds and that the Yamabe invariant of $M$ is non-negative. Then the Yamabe invariant of $M \times N$ is non-negative.

Proof: Given any $\epsilon>0$ we will show that there is a metric on $M \times N$ of unit volume and constant scalar curvature bounded below by $-\epsilon$. This implies the lemma.

Every compact smooth manifold admits a metric of constant scalar curvature. After rescaling, we can then pick a metric $g$ on $N$ of volume $V$ whose scalar curvature is a constant greater than $-\epsilon / 2$. The fact that the Yamabe invariant of $M$ is non-negative assures that there is a metric $h$ on $M$ of volume $1 / V$ and whose scalar curvature is a constant greater than $-\epsilon / 2$. 
The product metric $g+h$ on $N \times M$ has unit volume and scalar curvature bounded below by $-\epsilon$ as required.

We can now prove:

Theorem 2 : Every element in the spin cobordism group $\Omega_{n}^{\text {Spin }}$ can be represented by a connected spin manifold with non-negative Yamabe invariant.

Proof: The statement is trivial in the case $n=1$. When $n=2$, we have that $\Omega_{2}^{\text {Spin }}$ is isomorphic to $\mathbf{Z}_{2}$, and its two elements are represented respectively by a torus (the product of two copies of the spin structure on $S^{1}$ that is not a spin boundary), whose Yamabe invariant is zero, and by the 2-sphere with its canonical spin structure (the Yamabe invariant of the 2sphere is $8 \pi)$. When $n=3,5,6$ or 7 , the spin cobordism group $\Omega_{n}^{S p i n}$ is trivial, and its only element can be represented by the sphere of the corresponding dimension (which of course has positive Yamabe invariant). For $n=4$, the spin cobordism group is isomorphic to $\mathbf{Z}$ generated by the class of a $K 3$ surface. Note that the Yamabe invariant of the $K 3$ surface is zero, since it admits a scalar flat metric (the Ricci-flat metrics constructed by Yau [26]) but no metric of positive scalar curvature (since its $\hat{A}$-genus does not vanish). The same is true for the connected sum of any (positive) number of copies of the $K 3$ surface. The zero element in the group can be represented by the 4 -sphere.

Now recall that D.D. Joyce constructed examples of 8-dimensional compact Riemannian manifolds with holonomy $\operatorname{Spin}(7)$ (see [7]). These manifolds are then necessarily simply connected, spin, Ricci-flat and have $\hat{A}$-genus 1 (actually D.D. Joyce shows explicitly that his examples have $\hat{A}$-genus 1 , and use this to prove that they have holonomy $\operatorname{Spin}(7)$ and not a proper subgroup of it). Call $J_{8}$ one of these examples. Then $\alpha\left[J_{8}\right]$ is a generator of $K O_{8}(p t)$. Hence, multiplication by $\alpha\left[J_{8}\right]$ gives an isomorphism between $K O_{n}(p t)$ and $K O_{n+8}(p t)$. Note also that the Yamabe invariant of $J_{8}$ is zero (it can not be positive since the $\hat{A}$-genus is non-zero).

Now consider any class $[X] \in \Omega_{n}^{\text {Spin }}$ with $n \geq 8$. Let $P$ be a closed spin manifold of dimension $n-8$ so that $\alpha[P]$ is a generator of $K O_{n-8}(p t)(\alpha$ is an epimorphism in every dimension), and let $Q=P \times J_{8}$. Note that it follows from Lemma 1 that $Y(Q) \geq 0$. Note also that $\alpha[Q]$ is a generator of $K O_{n}(p t)$. There exists then an integer $k$ so that $\alpha[X]=k \alpha[Q]$. This means 
that $[X]-k[Q]$ is in the kernel of $\alpha$ and therefore, from Stolz's Theorem (see [22]), it can be represented by a closed connected spin manifold $S$ of strictly positive Yamabe invariant. Finally, $[X]$ can be represented by the manifold $\bar{X}$ obtained as the connected sum of $S$ and $k$ copies of $Q$. But the condition that the Yamabe invariant is non-negative is closed under connected sum (see [8]), and hence $Y(\bar{X}) \geq 0$. This completes the proof of Theorem 2 .

\section{Proof of Theorem 1 and final remarks}

We will now prove Theorem 1. We will use the following result of Gromov and Lawson [四, proof of Theorem B]:

Theorem 3 : Let $N$ be a compact simply connected spin n-dimensional manifold. Suppose that $n \geq 5$ and that the manifold $X$ is spin cobordant to $N$. Then $N$ is obtained from $X$ by doing surgery (on $X$ ) on spheres of codimension greater than two.

Let $M$ be a compact simply connected manifold of dimension at least five. It was proved by Gromov and Lawson in [4, that if $M$ is not spin then it admits a metric of positive scalar curvature. This means that the Yamabe invariant of $M$ is strictly positive. We can therefore assume that $M$ is spin.

We have proved in Theorem 2 that $M$ is spin cobordant to a spin manifold $X$ with non-negative Yamabe invariant. It follows from Theorem 3 that $M$ is obtained from $X$ by doing surgery on spheres of codimension greater than two. Finally, it is proved in [16] that if $\widehat{N}$ is obtained from the compact smooth manifold $N$ by doing surgery on spheres of codimension greater than two then $Y(\widehat{N}) \geq Y(N)$. Applying this result to our situation, we see that $Y(M) \geq Y(X) \geq 0$, and we have therefore finished the proof of Theorem 1 .

Remark: It is clear from Theorem 1 that for any simply connected compact manifold $X$ of dimension greater than four so that $\alpha[X] \neq 0$ the Yamabe invariant of $X$ is zero.

Remark: One of the main motivations for the study of the Yamabe invariant is the minimax method to construct Einstein metrics [21]. Assume for simplicity that $Y(X) \leq 0$. Then the Yamabe invariant of $X$ is the supremum of 
the scalar curvature over the family of metrics on $X$ of constant scalar curvature and unit volume. Moreover, if the supremum is achieved by a metric $g$ (as before), then $g$ is Einstein. Unfortunately, it is not always the case that the supremum is achieved. One can deduce for instance many examples from Theorem 1. Namely, if a simply connected manifold $X$ (compact of dimension greater than four) has non-vanishing $\alpha$-genus, then $Y(X)=0$. Hence the minimax procedure would provide a Ricci-flat metric. But, as it is wellknown since the work of Lichnerowicz, when the scalar curvature vanishes the Weitzenböck formula for the Dirac operator shows that every harmonic spinor is parallel. And the existence of non-trivial parallel spinors is a very restrictive condition (we know that in our examples there are non-trivial harmonic spinors since the $\alpha$-genus is not zero). It implies that our simply connected manifold must be the product of certain 8-dimensional manifolds and a Ricci-flat Kähler manifold (see [6, Theorem 1.2 and footnote p.54]). This, of course, implies that for most of the examples we consider the mini-

max method to construct an Einstein metric does not work. In particular, it does not work for any of the exotic spheres $S$ such that $\alpha[S] \neq 0$ (see [6, 14]).

Acknowledgements: The author would like to thank Claude LeBrun for many very useful observations related to this work. He would also like to thank the staff and directors of the Max Planck Institut for their hospitality during the preparation of this work and to Vyacheslav Krushkal and Ernesto Lupercio for many helpful discussions.

\section{References}

[1] M. Anderson, Scalar Curvature and Geometrization Conjectures for 3manifolds, Comparison Geometry, MSRI Publ. 30 (1996), 47-80.

[2] D.W. Anderson, E.H. Brown, F.P. Peterson, The structure of the spin cobordism ring, Ann. of Math. 86 (1967), 271-298.

[3] T. Aubin, Equations differentielles non lineaires et probleme de Yamabe concernant la courbure scalaire, J. Math. Pures Appl. 55 (1976), 269-296.

[4] M. Gromov, H. B. Lawson, The classification of simply connected manifolds of positive scalar curvature, Ann. Math. 111 (1980) 423-434. 
[5] M. Gursky, C. LeBrun, Yamabe Invariants and Spinc Structures, to appear in Geom. Func. An.

[6] N. Hitchin, Harmonic spinors, Adv in Math. 14 (1974), 1-55.

[7] D.D. Joyce, Compact 8-manifolds with holonomy Spin(7), Invent. math. 123 (1996), 507-552.

[8] O. Kobayashi, Scalar Curvature of a metric with Unit Volume, Math. Ann. 279 (1987), 253-265.

[9] H. B. Lawson, M.L. Michelson, Spin Geometry, Princeton University Press, 1987.

[10] C. LeBrun, Four-Manifolds without Einstein Metrics, Math. Res. Lett. 3 (1996), 133-147.

[11] C. LeBrun, Kodaira Dimension and the Yamabe Problem, to appear in Comm. An. Geom.

[12] C. LeBrun, Yamabe Constants and the Perturbed Seiberg-Witten Equations, Comm. An. Geom. 5 (1997), 535-553.

[13] A. Lichnerowicz, Spineurs harmoniques, C.R. Acad. Sci. Paris Ser. A-B, 257 (1963), 7-9.

[14] J. Milnor, Remarks concerning spin manifolds, Differential and Combinatorial Topology, a Symposium in Honor of Marston Morse, Princeton University Press, 1965, 55-62.

[15] T. Miyazaki, Simply connected spin manifolds and positive scalar curvature, Proc. A.M.S. 93 (1985), 730-734.

[16] J. Petean, G. Yun, Surgery and the Yamabe invariant, preprint.

[17] J. Petean, Computations of the Yamabe invariant, preprint.

[18] J. Rosenberg, $C^{*}$-algebras, positive scalar curvature, and the Novikov Conjecture III, Topology 25 (1986), 319-336.

[19] R. Schoen, S.T. Yau, On the structure of manifolds with positive scalar curvature Manuscr. Math. 28 (1979), 159-183. 
[20] R. Schoen, Conformal deformation of a Riemannian metric to constant scalar curvature, Journal Diff. Geom. 20 (1984), 479-495.

[21] R. Schoen, Variational Theory for the Total Scalar Curvature Functional for Riemannian Metrics and Related Topics, Lecture Notes in Math. 1365, Springer-Verlag, Berlin, (1987) 120-154.

[22] S. Stolz, Simply connected manifolds of positive scalar curvature, Ann. of Math. 136 (1992), 511-540.

[23] R. E. Stong, Notes on Cobordism Theory, Princeton University Press, 1968 .

[24] N.S. Trudinger, Remarks concerning the conformal deformation of Riemannian structures on compact manifolds, Ann Scuola Norm. Sup. Pisa 22 (1968), 265-274.

[25] H. Yamabe, On a deformation of Riemannian structures on compact manifolds, Osaka Math. J. 12 (1960), 21-37.

[26] S.T. Yau, On Calabi's conjecture and some new results in algebraic geometry, Proc. Nat. Acad. Sci. USA 74 (1977), 1798-1799. 